\title{
A NEW METHIONINE ANTAGONIST THAT HAS ANTIFUNGAL ACTIVITY: MODE OF ACTION
}

\author{
Yuhko Aoki, Masumi Kondoh, Mutsumi Nakamura, Toshriko Funi, \\ Toshikazu Yamazaki, Hisao Shimada and Mikio Arisawa \\ Nippon Roche Research Center, \\ 200 Kajiwara, Kamakura 247, Japan
}

(Received for publication January 14, 1994)

\begin{abstract}
A new antifungal, azoxybacilin (an unusual amino acid with an azoxy moiety) was identified from Bacillus cereus ${ }^{\dagger}$, and its in vitro antifungal activity and mode of action were investigated. Azoxybacilin was active against a broád spectrum of fungi. It was especially active against mycelial fungi, such as $A$ spergillus, and did not show antibacterial activity. No cross-resistance with antifungals currently on the market was observed.

The $\mathrm{IC}_{50}$ values of azoxybacilin antifungal activity against Saccharomyces cerevisiae were significantly greater when amino acids containing sulfur were added to the growth medium, whereas other amino acids were not effective at all. We, therefore, tested the effect of the intermediates involved in the synthetic pathway of these amino acids. The activity markedly diminished when one of the following four intermediates was present in the medium: homocysteine, cysteine, cystathionine or methionine. These four intermediates were the same as those required for the growth of the $O$-acetylhomoserine sulfhydrylase mutant, $S$. cerevisiae $\mathrm{ONO} 726$, indicating that azoxybacilin would inhibit a step or steps in the sulfur-fixation pathway.
\end{abstract}

A wide variety of antifungals effective in a systemic fungal infection model have been isolated, but only limited numbers of compounds have had their mode of action and the selectivity against fungi and mammals clarified. Among many natural antifungals, the following are representative selective antifungals: i) papulacandins ${ }^{2)}$, echinocandins ${ }^{3)}$, and acleacins ${ }^{4)}$ (inhibitors of $\beta$-(1,3)-D-glucan synthase); ii), nikkomycins ${ }^{5)}$ and polyoxins ${ }^{6)}$ (inhibitors of chitin synthases); iii) RI331 ${ }^{7)}$ (inhibitor of homoserine dehydrogenase); iv) benanomycin $\mathrm{A}^{8)}$ and $\mathrm{BMY}-28864^{93}$ (mannan-binding agents); v) soraphens ${ }^{10)}$ (inhibitors of acetyl-CoA carboxylase); vi) strobilurins-oudemansins ${ }^{11}$ (inhibitors of cytochrome-c oxidoreductase), and vii) Ro 09-1470 ${ }^{12 \sim 14)}$ (inhibitor of P450 lanosterol C-14 demethylase).

In the report which precedes this one ${ }^{1)}$, we describe on the isolation and structural elucidation of azoxybacilin (Fig. 1), a new antifungal of microbial origin, which has an unusual amino acid structure with an azoxy moiety. Here, we describe its in vitro antifungal activity and report on the results with respect to its mode of action. Azoxybacilin had a broad spectrum of antifungal activity. Its activity was antagonized by intermediates in the biosynthetic pathway of amino acids containing sulfur: homocysteine, cysteine, cystathionine and methionine, which were the same as those required for the growth of the $O$ acetylhomoserine sulfhydrylase mutant, Saccharo-

Fig. 1. The structure of azoxybacilin. myces cerevisiae ONO726. This results suggest that azoxybacilin would inhibit a step or steps in the sulfur-fixation pathway. We, thus, identified an antifungal that has a new mode of action.

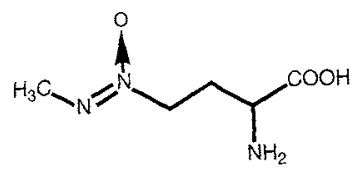

$\uparrow$ See ref 1 


\section{Materials and Methods}

\section{Materials}

Strains used for the measurement of in vitro antifungal activity were purchased from ATCC or IFO, or obtained as indicated in Table 1. S. cerevisiae ONO1 (prototroph) and S. cerevisiae ONO726 (methionine auxotroph) were generously provided by Dr. ONO, Okayama University, Okayama, Japan. The reference drugs, fluconazole (Pfizer), ketoconazole (Sigma), anisomycin (Sigma), tunicamycin (Sigma), and acleacin A (provided from Asahi Chemical Industry Co., Ltd., Tokyo) were used in a solution of dimethyl sulfoxide. 5-Flucytosine (obtained from F. Hoffman-La Roche AG., Basle), RI331 (provided from Taisho Co., Ltd., Tokyo), tetain (purified from Bacillus spp.), and amino acids were used in a water solution. $O$-acetylhomoserine and $O$-acetylserine were synthesized from homoserine and serine, respectively ${ }^{15}$, and the other amino acids were purchased from Sigma.

In Vitro Antifungal and Antibacterial Activities

In vitro antifungal activities were measured by the method described in a previous paper ${ }^{12\}}$. For this assay, YNB medium containing yeast nitrogen base without amino acid (Difco) $0.67 \%$, glucose $1 \%$ and $\mathrm{K}_{2} \mathrm{HPO}_{4} 0.25 \%$ was used instead of YNBAP, and for the measurement of antibacterial activities, M9 medium was used.

Measurement of Syntheses of Biomolecules

We measured the biosyntheses of DNA, RNA, protein, total lipids and ergosterol by methods described in a previous paper ${ }^{12)}$. Syntheses of cell wall components, glucan and mannan, were measured by using $\mathrm{D}-\left[U_{-}{ }^{14} \mathrm{C}\right]$ glucose (Amersham) ${ }^{16}$.

\section{Electron Microscopic Studies}

The cell surface of S. cerevisiae and Candida albicans were examined with a high-resolution scanning electron microscope (JEOL, JSM-35CF) ${ }^{17}$ ). The effect of azoxybacilin was compared with those of acleacin A, polyoxin D, and tetain. Germ-tube formation was examined with a light optical microscope (Olympus, BH2) after the incubation of C. albicans in $\mathrm{YNB}$ medium at $37^{\circ} \mathrm{C}$ in $5 \% \mathrm{CO}_{2}$ atmosphere, in which almost all of the control cells had converted to the hyphal form and ketoconazole-treatment inhibited the germ-tube formation.

\section{Results}

\section{In vitro Antifungal Activity}

Table 1 lists the in vitro antifungal activities of azoxybacilin, 5-flucytosine, fluconazole, and ketoconazole against 22 strains belonging to 7 clinically important species. Azoxybacilin had antimycotic activity against a broad spectrum of fungi, especially against mycelial fungi such as Absidia, Aspergillus, Microsporum and Trichophyton, but it was not active against Cryptococcus neoformans. When azoxybacilin was compared with the reference antifungals, 5-flucytosine, fluconazole and ketoconazole, it was more active against mycelial fungi than 5-flucytosine and fluconazole were. Azoxybacilin did not show any antibacterial activity against Escherichia coli and Bacillus subtilis.

We investigated the antifungal activity of azoxybacilin against strains resistant to azoles or 5-flucytosine or both. Azoxybacilin did not show cross-resistance with 5-flucytosine, fluconazole and ketoconazole (Table 2). Azoxybacilin did exhibit activity against a 5-flucytosine-resistant strain (C. albicans 652), an azoleresistant strain $(C$. albicans. $\mathrm{KB}$ ) and even against a 5-flucytosine and azole double resistant strain (C. albicans $\mathrm{AD}$ ), while the activities of 5-flucytosine and azole antifungals against the azoxybacilin-resistant strain C. albicans CY3003 were the same as those against C. albicans ATCC10231. The mode of action of azoxybacilin would be different from those of antifungals currently on the market. 
Table 1. Comparative antifungal activity of azoxybacilin with 5-flucytosine, fluconazole, and ketoconazole.

\begin{tabular}{|c|c|c|c|c|c|}
\hline \multirow{2}{*}{ Strains } & & \multicolumn{4}{|c|}{ Antifungal activity $\left(\mathrm{IC}_{80} \mu \mathrm{g} / \mathrm{ml}\right)$} \\
\hline & & Azoxybacilin & 5-Flucytosine & Fluconazole & Ketoconazole \\
\hline Candida albicans & ATCC 10231 & 4.2 & 0.031 & 0.99 & $<0.0004$ \\
\hline C. albicans & $652^{a}$ & 5.8 & $>200$ & 0.94 & $<0.0004$ \\
\hline C. glabrata & $975717^{\mathrm{a}}$ & 0.017 & 0.0051 & 3.5 & $<0.0004$ \\
\hline C. glabrata & $9895^{\mathrm{a}}$ & 0.022 & 0.0059 & 3.4 & 0.048 \\
\hline C. kefyr & IFO 0586 & 1.6 & 0.0038 & 0.23 & 0.0035 \\
\hline Cryptococcus albidus & IFO 0378 & 26 & $>200$ & 100 & 0.47 \\
\hline C. neoformans & MTU $13001^{\mathrm{b}}$ & $>200$ & 0.98 & 3.8 & $<0.0004$ \\
\hline C. neoformans & $422^{a}$ & $>200$ & $>200$ & 5.4 & $<0.0004$ \\
\hline Trichosporon beigelii & IFO 1198 & 0.054 & 2.9 & 0.18 & 0.0033 \\
\hline Absidia corymbifera & IFO 8084 & 5.7 & $>200$ & $>200$ & 0.26 \\
\hline Aspergillus fumigatus & MTU $06002^{\mathrm{b}}$ & 0.78 & 9.5 & 140 & 0.28 \\
\hline A. fumigatus & MTU $06006^{\mathrm{b}}$ & 0.72 & 5.4 & 200 & 0.53 \\
\hline A. fumigatus & MTU $06012^{\mathrm{b}}$ & 1.3 & 1.1 & $>200$ & 1.5 \\
\hline A. flavus & IAM 13835 & 9.8 & 3.2 & $>200$ & 0.38 \\
\hline A. flavus & CF $3002^{\circ}$ & 2.1 & 1.6 & 87 & 0.13 \\
\hline Microsporum canis & CF $1008^{\complement}$ & 0.055 & $>200$ & 28 & 0.05 \\
\hline M. gypseum & $\mathrm{Mi}-56^{\mathrm{d}}$ & 1.5 & $>200$ & 19 & 0.18 \\
\hline M. gypseum & IFO 8231 & 1.9 & $>200$ & 12 & 0.54 \\
\hline Trichophyton mentagrophytes & IFO 5971 & 0.24 & $>200$ & 17 & 0.089 \\
\hline T. mentagrophytes & IFO 7522 & 0.034 & $>200$ & 13 & 0.005 \\
\hline T. rubrum & IFO 5807 & 0.75 & $>200$ & 4.1 & $<0.0004$ \\
\hline T. rubrum & IFO 9185 & 1.1 & $>200$ & 0.96 & $<0.0004$ \\
\hline
\end{tabular}

Obtained from ${ }^{a}$ Hoffmann-La Roche, AG., Basle, Switzerland, ${ }^{b}$ Faculty of Medicine, University of Tokyo, Japan, ${ }^{\mathrm{c}}$ Kitasato University, Japan, ${ }^{\mathrm{d}}$ National Institute of Hygienic Science, Tokyo, Japan.

Table 2. Comparative antifungal activity of azoxybacilin with 5-flucytosine, fluconazole, and ketoconazole against C. albicans resistant strains.

\begin{tabular}{|c|c|c|c|c|}
\hline \multirow{2}{*}{ Strains } & \multicolumn{4}{|c|}{ Antifungal activity $\left(\mathrm{IC}_{80} \mu \mathrm{g} / \mathrm{ml}\right)$} \\
\hline & Azoxybacilin & 5-Flucytosine & Fluconazole & Ketoconazole \\
\hline Candida albicans ATCC 10231 & $4.2(1)^{\mathfrak{c}}$ & 0.031 & $0.99(1)$ & $<0.0004$ \\
\hline C. albicans $652^{\mathrm{a}}$ & $5.8(1)$ & $>200 \quad(>6,400)$ & $0.94(1)$ & $<0.0004$ \\
\hline C. albicans $\mathrm{AD}^{\mathrm{a}}$ & $4.1(1)$ & 0.11 & $34 \quad(34)$ & 0.16 \\
\hline C. albicans $\mathrm{KB}^{\mathbf{a}}$ & $17 \quad(4)$ & $>200 \quad(>6,400)$ & $(36)$ & $0.015(>400)$ \\
\hline C. albicans CY $3003^{\mathrm{b}}$ & $83 \quad(20)$ & 0.049 & 1.0 ( 1$)$ & $<0.0004 \quad(1)$ \\
\hline
\end{tabular}

Obtained from ${ }^{a}$ Kitasato University, Japan, ${ }^{b}$ Hoffmann-La Roche, AG., Basle, Switzerland. ${ }^{c}$ Relative values by setting each susceptibility of ATCC 10231 as one.

\section{Effects on Biomolecule Syntheses and Morphology}

Effects of azoxybacilin on the biosyntheses of DNA, RNA, protein, mannan, glucan, total lipids and ergosterol were compared with those of antifungal compounds whose mode of actions have already been clarified. While 5-flucytosine, acleacin A, tunicamycin, and ketoconazole specifically inhibited nucleic acids-, glucan-, mannan-, and ergosterol-biosyntheses, respectively, azoxybacilin $(1.36 \mu \mathrm{g} / \mathrm{ml})$ and $\mathrm{RI} 331$ $(7.72 \mu \mathrm{g} / \mathrm{ml})$ were only able to show scattered inhibition of the molecules (Fig. 2). The similar inhibition profiles by both azoxybacilin and RI331 were observed at any concentration.

For the electron microscopy studies, azoxybacilin did not affected the external view of the cell surface as the cell wall inhibitors, acleacin $\mathrm{A}$, polyoxin $\mathrm{D}$, and tetaine ${ }^{7)}$ changed the cell surface of $S$. cerevisiae 
Fig. 2. Effects of antifungal compounds on biomolecular syntheses in $S$. cerevisiae.

A) Azoxybacilin, $1.36 \mu \mathrm{g} / \mathrm{ml}$, B) 5-flucytosine, $6.7 \mu \mathrm{g} / \mathrm{ml}$, C) RI331, $7.72 \mu \mathrm{g} / \mathrm{ml}$, D) acleacin A, $0.56 \mu \mathrm{g} / \mathrm{ml}$, E) tunicamycin, $0.67 \mu \mathrm{g} / \mathrm{ml}$, and F) ketoconazole, $6.7 \mu \mathrm{g} / \mathrm{ml}$.
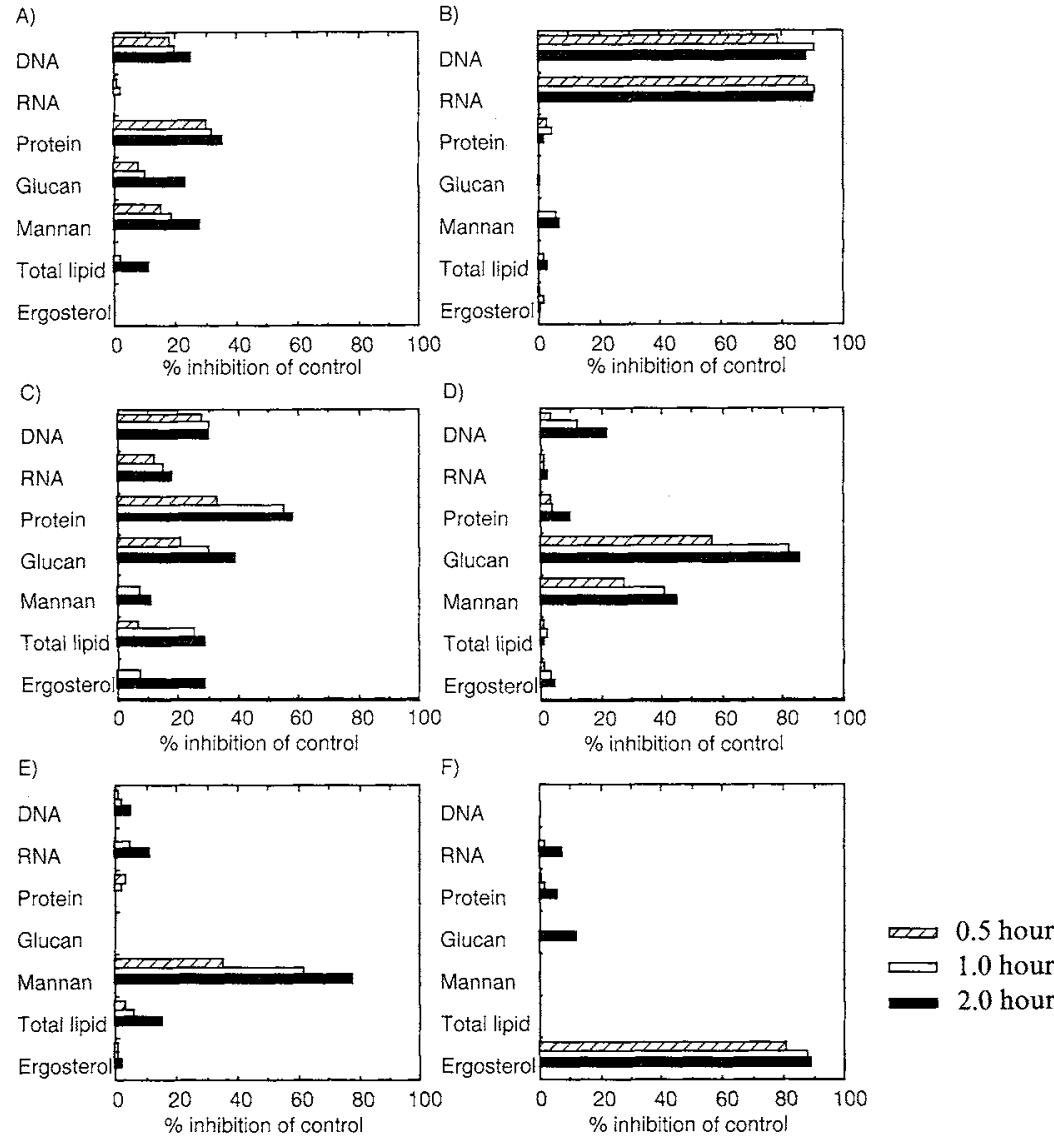

and $C$. albicans from smooth-walled and spherical to profoundly retracted and distorted (data not shown). This suggests that the mode of action of azoxybacilin is different from these compounds. On the other hand, azoxybacilin inhibited the germ-tube formation of $C$. albicans with an $\mathrm{IC}_{50}$ of $1.2 \mathrm{mg} / \mathrm{ml}$.

All of the results indicated that the target sits of azoxybacilin is not involved in biomolecular syntheses of nucleic acids, protein, glucan, mannan, chitin and ergosterol.

\section{Effects of Amino Acids on the Antifungal Activity}

Since azoxybacilin was structurally thought to be an amino acid analog, and since the inhibition profile of its biomolecular syntheses was similar to that of RI331, an inhibitor of homoserine dehydrogenase which catalyzes the biosynthesis of amino acids containing sulfur (Fig. 3$)^{7}$, we expected azoxybacilin to be an amino acid biosynthesis inhibitor. We, therefore, examined the antagonistic effect of individual amino acids on the antifungal activity of azoxybacilin against $S$. cerevisiae. The corresponding $\mathrm{IC}_{50}$. values are listed in Table 3. The antifungal activity of RI331 was affected by the presence of homoserine, methionine, cysteine, serine, threonine, or isoleucine which are all involved in a large family of sulfurcontaining amino acid biosyntheses (Fig. 3). This is consistent with the fact that RI331 inhibits homoserine 
Fig. 3. Biosyntheses of methionine and cysteine in S. cerevisiae.

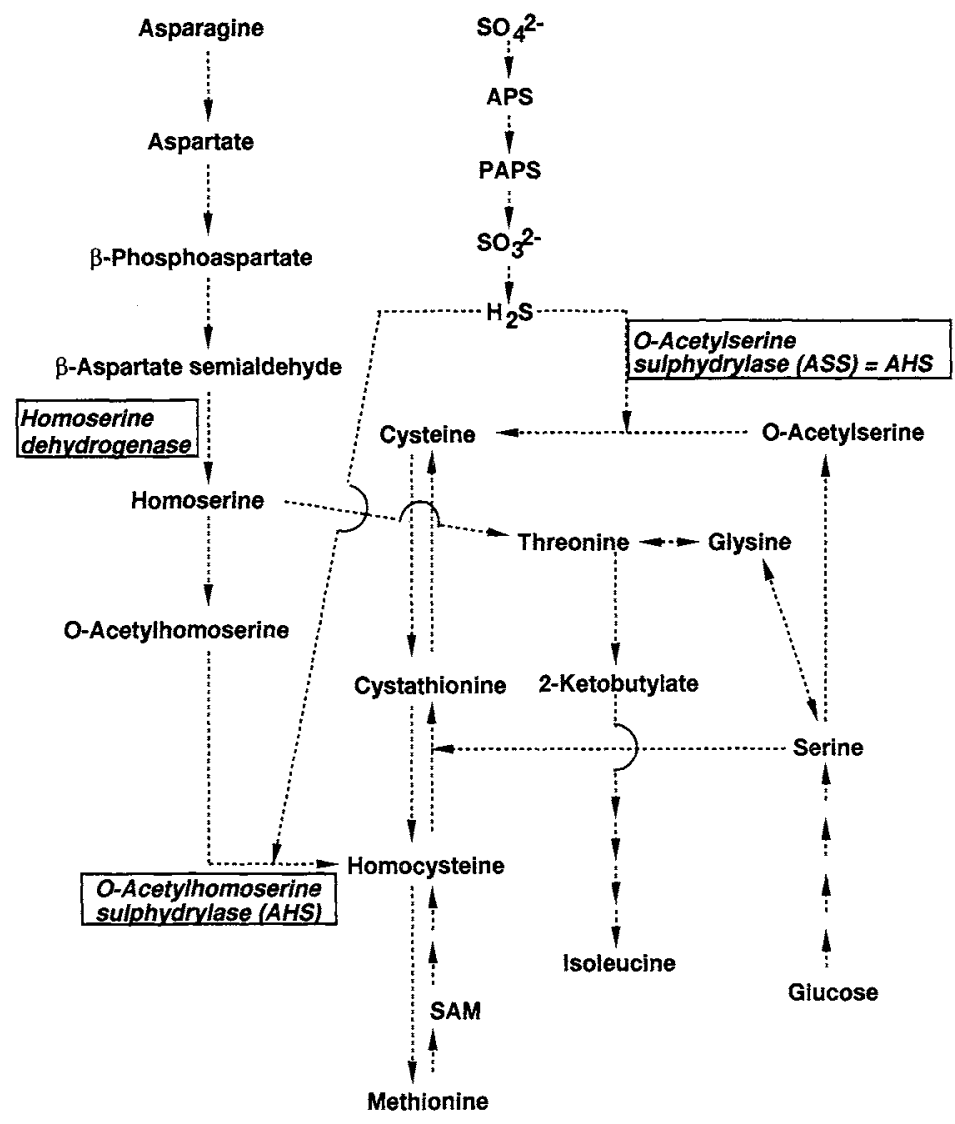

Table 3. Antagonistic effects of amino acids on the antifungal activity of azoxybacilin and RI331 against $S .{ }^{\circ}$ cerevisiae ONO1.

\begin{tabular}{|c|c|c|c|c|c|}
\hline \multirow{2}{*}{$\begin{array}{l}\text { Amino acids } \\
\text { added to } \\
\text { minimum medium }\end{array}$} & \multicolumn{2}{|c|}{ Relative antifungal activities } & \multirow{2}{*}{$\begin{array}{l}\text { Amino acids } \\
\text { added to } \\
\text { minimum medium }\end{array}$} & \multicolumn{2}{|c|}{ Relative antifungal activities } \\
\hline & Azoxybacilin & RI331 & & Azoxybacilin & RI331 \\
\hline Arginine & 1.9 & 2.0 & Lysine & 1.6 & 0.8 \\
\hline Asparagine & 1.6 & 1.8 & Alanine & 1.4 & 1.0 \\
\hline Homoserine & 2.1 & 44 & Glutamate & 0.7 & 0.5 \\
\hline Methionine & 101 & 17 & Glutamine & 1.2 & 0.9 \\
\hline Cysteine & 24 & 5.8 & Histidine & 1.1 & 1.2 \\
\hline Serine & 1.3 & 3.8 & Phenylalanine & 1.1 & 0.8 \\
\hline Threonine & 1.7 & 3.3 & Tryptophan & 1.2 & 1.1 \\
\hline Isoleucine & 1.7 & 4.2 & Tyrosine & 0.8 & 0.6 \\
\hline Leucine & 0.6 & 0.5 & Proline & 1.0 & 1.3 \\
\hline Valine & 0.4 & 0.4 & None & 1.0 & 1.0 \\
\hline Glysine & 1.4 & 1.0 & & & \\
\hline
\end{tabular}

dehydrogenase which catalyze the conversion from $\beta$-aspartate semialdehyde to homoserine. On the contrary, $\mathrm{IC}_{50}$ values of azoxybacilin were significantly greater when $S$. cerevisiae ONO1 was grown in minimum medium with methionine or cysteine, while the other amino acids did not have the same effect. 
Table 4. Antagonistic effects of the intermediates of amino acids containing sulfur on the antifungal activity of azoxybacilin.

\begin{tabular}{|c|c|c|c|}
\hline \multirow{3}{*}{$\begin{array}{l}\text { Intermediates added } \\
\text { to minimal medium }\end{array}$} & \multicolumn{3}{|c|}{ Cell density $\left(\mathrm{OD}_{660} \times 1000\right)$} \\
\hline & \multicolumn{2}{|c|}{$S$. cerevisiae ONO 1} & \multirow{2}{*}{$\frac{\text { S. cerevisiae ONO } 726}{\text { - Azoxybacilin }}$} \\
\hline & -Azoxybacilin & $\begin{array}{c}+ \text { Azoxybacilin } \\
(7.8 \mu \mathrm{g} / \mathrm{ml})\end{array}$ & \\
\hline None & 235 & 2 & 0 \\
\hline Aspartate & 317 & 0 & 12 \\
\hline Asparagine & 324 & 5 & 13 \\
\hline Homoserine & 315 & 34 & 7 \\
\hline Serine & 305 & 0 & 0 \\
\hline$O$-Acetylhomoserine & 303 & 0 & 3 \\
\hline$O$-Acetylserine & 239 & 0 & 0 \\
\hline Homocysteine & 277 & 214 & 223 \\
\hline Cysteine & 193 & 159 & 237 \\
\hline Cystathionine & 303 & 142 & 252 \\
\hline Methionine & 282 & 285 & 253 \\
\hline Threonine & 285 & 4 & 0 \\
\hline Glycine & 341 & 23 & 6 \\
\hline Isoleucine & 290 & 10 & 0 \\
\hline
\end{tabular}

This result suggests that azoxybacilin would inhibit an enzyme or enzymes involved in the biosyntheses of only two of the sulfur-containing amino acids, methionine and cysteine; therefore, we propose that the target site is most likely different from that of RI331.

Effects of the Intermediates of the Biosyntheses of Amino Acids Containing Sulfur on the Antifungal Activity

Fig. 2 shows the metabolic map of methionine and cysteine biosyntheses in $S$. cerevisiae ${ }^{18}$ ). To know more about the exact target sites of azoxybacilin, we investigated the antagonistic effects of the amino acids involved in this pathway using the same type of experiment as that indicated in Table 3 . As shown in Table 4, its activity against $S$. cerevisiae was markedly diminished when either one of the following four intermediates: homocysteine, cysteine, cystathionine or methionine was present in the medium, whereas the addition of any of the other intermediates did not affect the activity of azoxybacilin at all. This allows us to conclude that azoxybacilin probably inhibits an enzyme or enzymes in the sulfur-fixation pathway from inorganic sulfate to cysteine or homocysteine. To confirm this, we designed experiments using a mutant strain lacking an enzyme for the sulfur-fixation pathway; it is S. cerevisiae ONO726 (derived from $S$. cerevisiae $\mathrm{ONO1}$ ) lacking $O$-acetylhomoserine sulfhydrylase (AHS) which catalyzes two reactions in $S$. cerevisiae: one is the conversion from $O$-acetylhomoserine to homocysteine and the other is the conversion of $O$-acetylserine to cysteine. The growth of the mutant was supported by the same set of amino acids that antagonized the action of azoxybacilin in the wild type strain (Table 4).

\section{Discussion}

From Bacillus cereus, we identified an antifungal agent, azoxybacilin, which is structurally new and has a broad spectrum of antifungal activity. The compound was suggested to exert antifungal activity by inhibiting a step or steps in sulfur-fixation on the basis of the following experimental results: i) the antifungal activity of azoxybacilin was antagonized by the intermediates of the biosynthetic pathway for amino acids containing sulfur: homocysteine, cysteine, cystathionine or methionine, and ii) the growth of a mutant 
lacking $O$-acetylhomoserine sulfhydrylase (an enzyme catalyzing the incorporation of organic sulfur into an amino acid) was supported by the same amino acids that showed antagonism to azoxybacilin.

Germ-tube formation was also inhibited by azoxybacilin. So far, two types of ergosterol inhibitors, azoles and allylamines have been reported to inhibit this formation ${ }^{19}$. We proved that azoxybacilin did not inhibit ergosterol biosynthesis as a primary target in the experiment of biomolecular synthesis (Fig. 2). The causes of this phenomenon, therefore, may be other than the ergosterol biosynthesis inhibition. There have been many reports on methionine as a modifier of the conversion to the mycelial form of C. albicans ${ }^{20)}$. It suggests that the inhibition of methionine biosynthesis may account for its inhibition of the germ-tube formation.

There have been no reports on the identification of antifungals that inhibit the sulfur-fixation either from a natural source or from synthetic products. For the first time, we demonstrate that an antifungal agent that can inhibit a step or steps in the sulfur-fixation pathway, dose indeed exist.

\section{Acknowledgment}

We wish to thank Dr. ONo of Okayama University for the valuable discussions as well as his generous supply of the strains. Thanks are also due to Dr. Oomura of Taisho Co., Ltd., for his generous supply of RI331.

\section{References}

I) Fuju, M.; S. Sawairi, H. Shimada, H. Takaya, Y. Aoki, T. OKuda \& K. Yokose: Azoxybacilin, a novel antifungal agent produced by Bacillus cereus NR2991. Production, isolation and structure elucidation. J. Antibiotics 47: $833 \sim 835,1994$

2) Duran, A.; R. VÁrona, P. Perez \& I. Garcia-Acha: Biosynthesis of fungal wall $\beta$-glucan and its inhibition by antifungal agents. In Microbial cell wall synthesis and autolysis. Ed., C. NombELA, Elsevier Science, Amsterdam, 1984

3) Sawistowska-Schröder, E. T.; D. Kerridge \& H. Perry: Echinocandin inhibition of I,3- $\beta$-glucan synthase from Candida albicans. FEBS. 173: 134 138, 1984

4) Yamaguchi, H.; T. Hiratani, K. Iwata \& Y. Yamamoto: Studies on the mechanism of antifungal action of aculeacin A. J. Antibiotics 35: 210 219, 1982

5) CABIB, E.: Differential inhibition of chitin synthetases 1 and 2 from Saccharomyces cerevisiae by polyoxin D and nikkomycins. Antimicrob. Agents Chemother. 35: 170 173, 1991

6) ENDO, A.; K. KaKiKi \& T. Misato: Mechanism of action of the antifungal agent polyoxin D. J. Bacteriol. 104: $189 \sim 196,1970$

7) Yamaki, H.; M. Yamaguchi, T. NishimuRA, T. Shinoda \& H. Yamaguchi: Unique mechanism of action of an antifungal antibiotic RI-331. Drug Exptl. Clin. Res. 14: 467 472, 1988

8) IKeda, D.; T. Nishizuka, S. P. Huang, S. Kondo \& T. Takeuchi: Amino acid analogs of benanomicin A through desalaninebenanomicin A. J. Antibiotics 45: 1645 1652, 1992

9) Sawada, Y.; K. Numata, T. Murakami, H. Tanimichi, S. Yamamoto \& T. Oki: Calcium-dependent anticandidal action of pradimicin A. J. Antibiotics 43: 715 721, 1990

10) Vahlensieck, H. F.; L. Pridzun, H. Reichendach \& A. Hinnen: Identification of the yeast ACCl gene product (acetyl-CoA carboxylase) as the target of the polyketide fungicide soraphen A. Curr. Genet. (Germany). 25: $95 \sim 100,1994$

11) Link, T. A.; U. HAase, U. Brandt \& G. Vonjagow: What information do inhibitors provide about the structure of the hydroquinone oxidation site of ubihydroquinone-cytochrome-C oxidoreductase. J. Bioenerg. Biomembranes. 25: $221 \sim 232,1993$

12) Aoki, Y.; T. Yamazaki, M. Kondoh, Y. Sudoh, N. Nakayama, Y. Sekine, H. Shimada \& M. Arisawa: A new series of natural antifungals that inhibit P450 lanosterol C-14 demethylase. J. Antibiotics 45: 160 170, 1992

13) Schwartz, R. E.; C. Dufresne, J. E. Flor, A. J. Kempf, K. E. Wilson, T. Lam, J. Onishi, J. Milligan, R. A. Fromtling, G. K. Abruzzo, R. Jenkins, K. Glazomitsky, G. Bills, L. Zitano, S. M. d. Val \& M. N. Omstead: Restriction, a novel glycine-containing antifungal agent. J. Antibiotics 44: 463 471, 1991

14) Aoki, Y.; F. Yoshihara, M. Kondoh, Y. Nakamura, N. Nakayama \& M. Arisawa: Ro 09-1470 is a selective inhibitor of P450 lanosterol C-14 demethylase of fungi. Antimicrob. Agents Chemother. 37: 2662 2667, 1993

15) NaGaI, S. \& M. Flavin: [195c] Synthesis of o-acetylhomoserine. In Method in enzymology. Metabolism of amino acids and amines. Ed., H. TABOR \& C. W. TABOR, pp. 423 424, Academic Press, New York and London, 1971

16) Dominguez, A.; R. M. Varona, J. R. Villanueva \& R. Sentandreu: Mutants of Saccharomyces cerevisiae cell 
division cycle defective in cytokinesis. Biosynthesis of the cell wall and morphology. Antonie van Leeuwenhoek. 48: $145 \sim 157,1982$

17) Yamaguchi, H.; T. Hiratani, M. Baba \& M. Osumi: Effect of aculeacin A a wall-active antibiotic, on synthesis of the yeast cell wall. Microbiol. Immunol. 29: 609 623, 1985

18) ONO, B.; Y. ShIRAHIGE, A. NaIJOH, N. ANDOH, H. OHUE \& Y. Ishino-Arao: Cysteine biosynthesis in Saccharomyces cerevisiae; mutation that confers cystathionine $\beta$-synthase deficiency. J. Bacteriol. 170: $5883 \sim 5889,1988$

19) Shimokawa, O.; Y. Kato and H. Nakayama: Accumulation of 14-methyl sterols and defective hyphal growth in Candida albicans. J. Med. Vet. Mycol. 24: 327 336, 1986

20) Yamaguchi, H., M. MiYail \& K. Nishura: Dimorphism. In Biology of Pathogenic Fungi. Ed., H. Yamaguchi, pp. 139 162, Nanzando, Tokyo, 1987 